\title{
Una nota a Tucídides I 22, 2
}

\author{
Ignacio Rodríguez Alfageme \\ Universidad Complutense de Madrid \\ iralfageme@ucm.es
}

Recibido: $30-11-2015$

Aceptado: 21-12-2015

\section{RESUMEN}

Este trabajo estudia las distintas interpretaciones del inicio del capítulo 22 centrándose en la segunda frase. Se

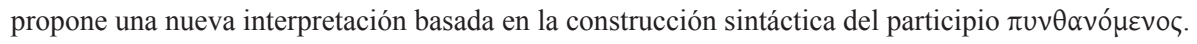

Palabras clave: Tucídides, método, antítesis.

\begin{abstract}
This paper examines the different interpretations of the beginning of chapter 22 focusing on the second sentence. A new interpretation based on the syntactic construction of the participle $\pi \cup v \theta \alpha v$ ó $\mu \varepsilon v o \varsigma$ is proposed.
\end{abstract}

Keywords: Thucydides, method, antithesis.

El principio del capítulo 22 del libro I de la Historia ha dado motivo a una larga serie de trabajos ${ }^{1}$ que tratan de desentrañar y precisar el significado de las palabras de Tucídides; la gran mayoría de ellos se centran en las dos primeras frases, donde se explica el modo como se han compuesto los discursos. La discusión está plenamente justificada, ya que se trata de la declaración del autor sobre el método ${ }^{2}$ que ha seguido al escribir la Historia. En este trabajo dejamos de lado la primera frase, la que se refiere a los discursos, aunque tendremos que hacer alusiones a ella, para centrarnos en

${ }^{1}$ Entre otros muchos vid. Schadewaldt (1929), Grosskinsky (1936), Pohlenz (1936), Gomme (1937), Harrison (1937), Finley (1939), Schmid (1954), Wille (1965), Luschnat (1973), Golan (1976-77), Loriaux (1982), Dover (1988), Hornblower (1988: 59-60), Iglesias Zoido (1989), Marincola (1989), Bicknell (1990), Garrity (1998), Tsakmakis (1998), Bonelli (2003).

${ }^{2}$ Incluso en este punto hay discrepancia de opiniones entre quienes presuponen que se trata de una afirmación programática, escrita antes del resto de la Historia, y quienes piensan lo contrario (vid. Harrison (1937: 7). El panorama de las distintas interpretaciones de esta frase anteriores a Grosskinsky (Steup, Pohlenz, Schadewaldt, Kapp) queda recogido en su obra (1936: 25-29); para las obras posteriores puede verse Finley (1942: 74-110 y 267-274), West (1973), Wilson (1982) y Swain (1993), entre otros. 
la que le sigue, que está en claro contraste con ella. El pasaje en su totalidad, según el texto de Alberti (1972) ${ }^{3}$, es el siguiente ${ }^{4}$ :

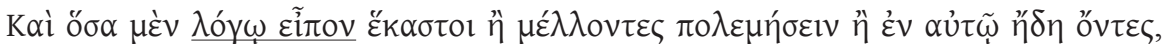

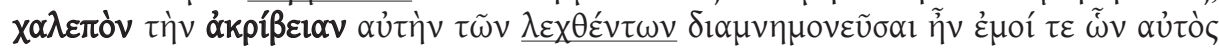

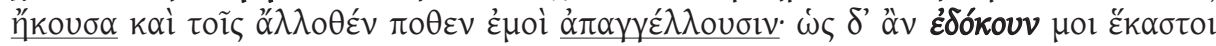

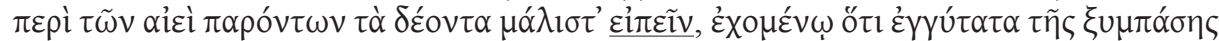

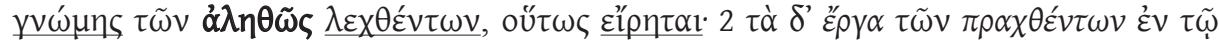

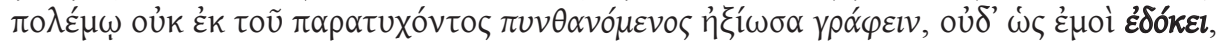

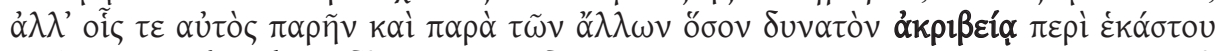

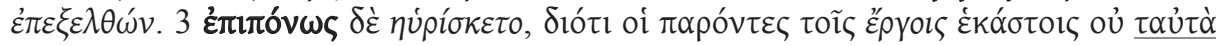

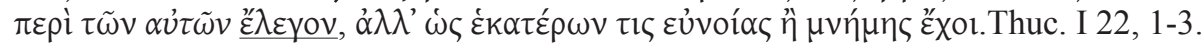

Los problemas que vamos a discutir se centran en cuatro puntos:

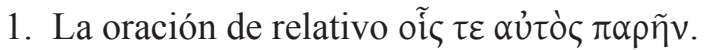

2. El valor de la coordinación $\tau \varepsilon \ldots$ koú.

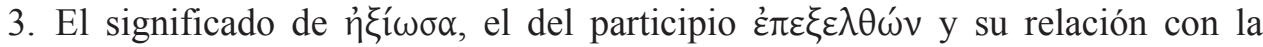
oración de relativo precedente.

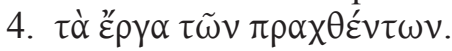

1. El texto de la segunda frase (a partir de $\alpha \lambda \lambda \lambda^{\prime}$ oí $\zeta$ ), referente a los hechos de guerra, plantea problemas de difícil solución; de ahí las correcciones de Linwood ( $\pi \varepsilon \rho i ́)$ y Ullrich, al que siguen Classen, Steup ${ }^{5}$ y Gomme, quien propone $<\tau \dot{\alpha}>\pi \alpha \rho \grave{\alpha}$ $\tau \tilde{\omega} \nu \stackrel{\alpha}{\lambda} \lambda \lambda \omega \nu$. La razón para enmendar el texto así se encuentra en la construcción del

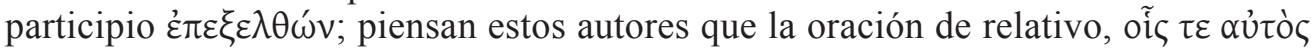
$\pi \alpha \rho \tilde{\eta} v$, es complemento directo del participio, «indagando las acciones de guerra en las que estuve presente en persona y a partir de otros... ${ }^{6}$, y en ese caso el segundo miembro introducido por koú cumpliría la misma función, de forma que la inclusión del artículo $<\tau \dot{\alpha}>$ para sustantivar $\pi \alpha \rho \dot{\alpha} \tau \tilde{\omega} \nu$ ó $\lambda \lambda \omega \nu$ haría la sintaxis menos abrupta al evitar la coordinación de un complemento preposicional con una oración de relativo. Pero lo ponen en duda los análisis de Wille (1965: 702-703), que la rechaza por estar basada exclusivamente en consideraciones de contenido sin tener en cuenta la forma, por un lado, y de Grosskinsky (1936: 102-104), por otro, que se basa en los

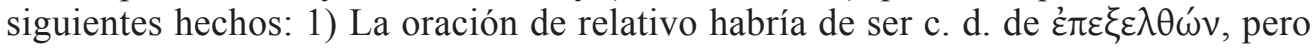

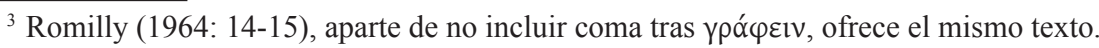

${ }^{4}$ Aparecen subrayados los términos que se refieren al discurso, en negrita, los que aluden a la exactitud del historiador, en cursiva, los relacionados con los hechos y en negrita cursiva los que hablan de la opinión del autor.

${ }^{5}$ Quien ofrece la siguiente traducción: «sondern in der Weise, dass ich sowohl die Dinge, bei denen ich persönlich zugegen war, als dasjenige, was mir von den anderen berichtet wurde, soviel wie möglich, den genauen Sachverhalt hinsichtlicht jeder Einzelheit nach erforscht hatte». A lo que añade «auch das Selbsterlebte erforderte, zumal bei wichtigen Vorgängen, genaue Untersuchung» (Classen - Steup, 1966: 78 y el comentario que se encuentra en pp. 408-409). Sobre esta conjetura vid. el resumen de Schmid (1954: 221-223).

${ }^{6}$ Macía (1989: 61) sigue esta linea de interpretación. 
es imposible que el término coordinado con ella ( $\pi \alpha \rho \alpha \grave{~} \tau \tilde{\omega} v$ ó $\lambda \lambda \omega v)$ pueda serlo (de ahí la corrección de Ullrich), 2) resulta inverosímil que Tucídides investigara sobre acontecimientos de los que ha sido testigo presencial, 3) el participio y sus comple-

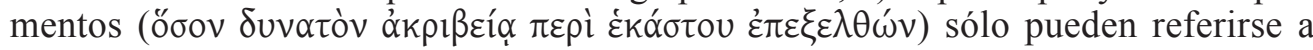
$\pi \alpha \rho \alpha ̀ \tau \tau \tilde{\omega} v$ ó $\lambda \lambda \omega v$. La otra solución posible coordina la oración de relativo y el par-

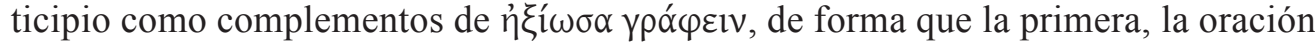
de relativo, sería equivalente a $\pi \alpha \rho \omega ́ v$. Y a partir del trabajo de Grosskinsky se impone en las ediciones y traducciones esta segunda interpretación (Romilly, Rodríguez Adrados, Torres Esbarranch); así Maddalena mantiene el texto transmitido sin hacer depender oĩ $\varsigma$ de $\dot{\varepsilon} \pi \varepsilon \xi \varepsilon \lambda \theta \omega ́ v$, remitiendo a Grosskinsky para afirmar: «porque no parece comprensible que Tucídides investigara sobre los hechos que conocía de primera mano». Pero, ni Grosskinsky ni Maddalena mencionan el hecho de que estos dos elementos se encuentran unidos mediante el juego de partículas $\tau \varepsilon \ldots$ kó́..., lo que plantea el problema de nuevo, ya que en el caso de la primera interpretación resulta difícil admitir que se unan ambos términos con esta combinación de partículas, como subraya Steup (1966: 408)7.

2. El problema, por lo tanto, lo podemos reducir en un primer momento a la interpretación que haya de darse a la aparición de $\tau \varepsilon .$. Koí en este lugar.

Separando en renglones aparte las oraciones encontramos la siguiente estructura del período en el que se inserta esta combinación'

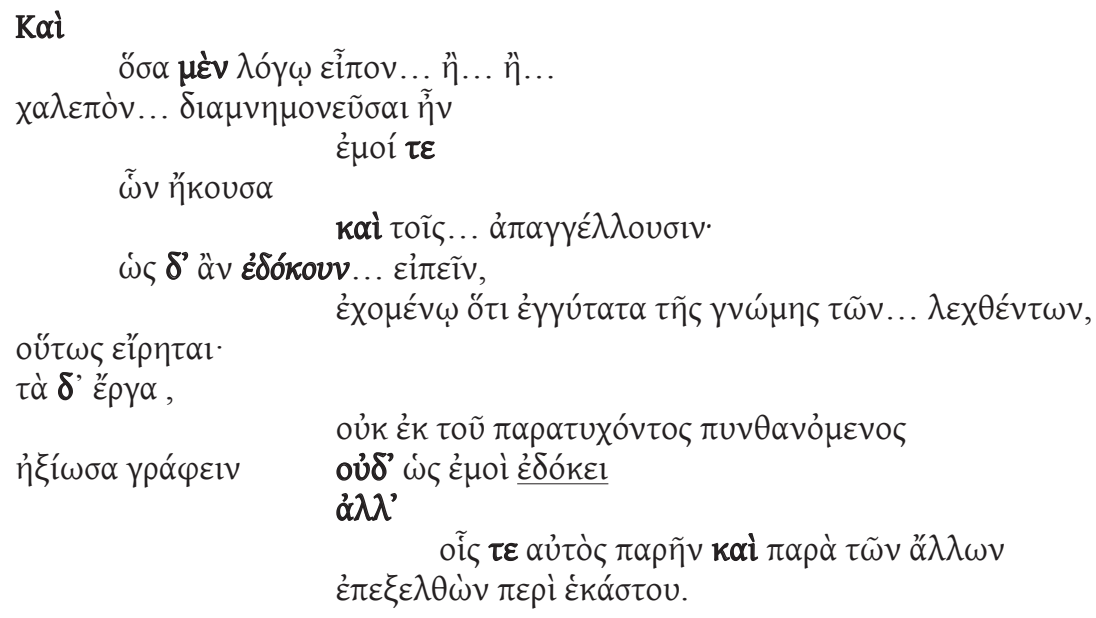

Es decir, de un modo más esquemático, y dejando de lado la conjunción kaí, que introduce el período en su conjunto, encontramos lo siguiente:

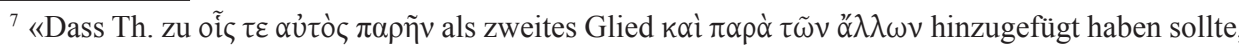
ist unmöglich.»

${ }^{8}$ Sobre la estructura de esta frase vid. Wille (1965: 700-716).
} 
$\chi \alpha \lambda \varepsilon \pi \grave{v} v \tilde{\eta} v$

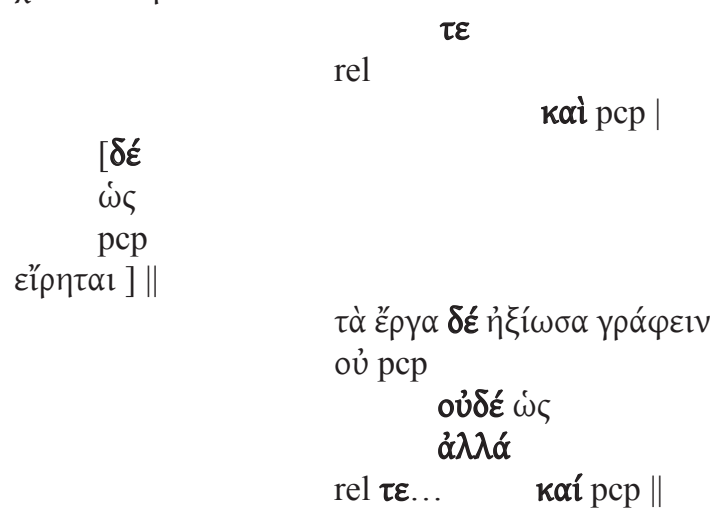

El párrafo está compuesto por tres oraciones complejas que desarrollan dos temas, discursos y acciones de guerra, según la antítesis bien conocida en Tucídides entre

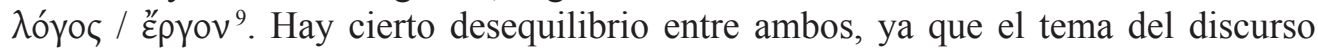
ocupa dos oraciones y el de las acciones solo una, pero sin duda este desequilibrio se organiza para colocar en el centro lo más destacado (y problemático) ${ }^{10}$, es decir, cómo se han compuesto los discursos dejando sentado que se ha hecho atendiendo a la $\delta$ ó $\alpha$

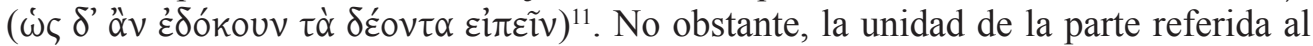
$\lambda$ خó os se deja bien establecida mediante los dos verbos de lengua que abren y cierran

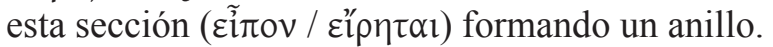

El paso a las acciones de guerra cambia por completo la postura del historiador; no sólo se prescinde de la $\delta$ ó $\alpha$ y de cualquier informante ocasional ( $\pi \alpha \rho \alpha \tau u \chi o ́ v \tau o \varsigma)$, sino

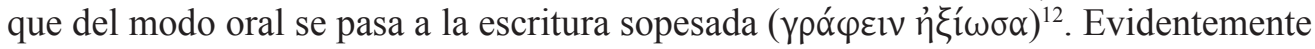
estas palabras no pueden tomarse en su sentido literal, pero creo que sirven claramente para marcar la distancia entre el modo de composición de los discursos y las acciones de guerra ${ }^{13}$. Así, dejando de lado la frase central, la comparación de la primera y la última resalta los paralelos de construcción y el contraste de tratamiento de los discursos frente a las acciones de guerra.

\footnotetext{
${ }^{9}$ Cf. Parry (1981), Tsakmakis (1998: 243).

${ }^{10}$ Para su interpretación vid. Schmid (1954), Loriaux (1982: 289-292), Iglesias Zoido (1989), Garrity (1998).

${ }^{11}$ Sobre este elemento subjetivo vid. Pohlenz (1936: 65-66).

${ }^{12}$ El significado de ả $\xi_{1}$ ó $\omega$ resulta complejo por las distintas acepciones con las que lo usa Tucídides, vid. Huart (1968: 247-249); en él está siempre presente el valor del adjetivo ớłıৎ del que deriva; de ahí acepciones como «considerar justo», «reclamar», «juzgar digno», «considerar», «exigir», «querer», «afirmar». Huart no comenta este ejemplo, pero, de acuerdo con la etimología que acepta Chantraine (1968: 94), hay que interpretarlo con un significado relacionado con la valoración que se logra mediante la acción de pesar algo, tal como hemos traducido.

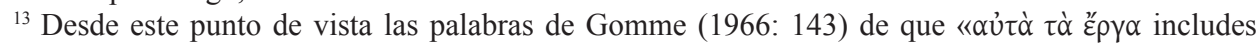

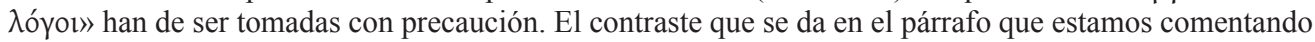
no permite aceptarla sin más al pie de la letra.
} 
El paralelo que se da entre la primera y la última frase invita a pensar que $\tau \varepsilon \ldots$ koí tiene el mismo valor en ambos lugares: la dificultad de recordar los discursos afecta tanto a Tucídides ( $\tau \varepsilon)$, como ( $\kappa \alpha i ́)$ a los demás testigos de los que se sirvió. Es decir, la combinación de partículas señala los dos extremos a los que el autor recurre, un

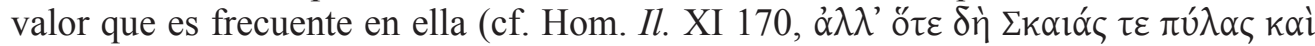

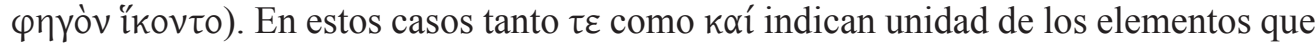
coordinan con la nota de que kái señala que el segundo elemento tiene que combinarse con el primero (Ruijgh, 1971: 130 y 171) y éste resalta en el conjunto (Ruijgh, 1971: 182). En nuestro pasaje los elementos unidos por $\tau \varepsilon \ldots$ koí en ambas frases tienen la misma estructura sintáctica: una oración de relativo en la primera parte y un participio en la segunda. A pesar de que el primer participio está sustantivado y el segundo no, esta coincidencia no puede ser casual. Si llevamos el paralelo a las dos oraciones de relativo, se ve claro que la primera es complemento del predicado principal

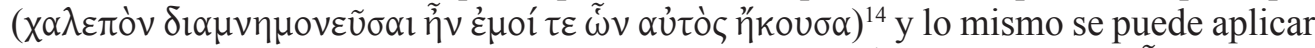

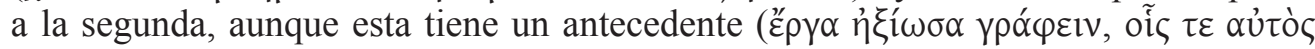
$\pi \alpha \rho \tilde{\eta} \nu)$, que falta en la primera. Así que, de seguir este paralelo, quedaría excluida la interpretación que hace depender la oración de relativo del participio $\dot{\pi} \pi \xi \xi \varepsilon \lambda \theta \omega ́ v$, aunque no se puede olvidar el hecho de que $\tau \varepsilon . .$. koí une miembros iguales para formar un todo. Además este tipo de coordinación entre elementos dispares no es raro en Tucídides; ninguno de los comentarios que he consultado hace mención de ellos, pero en el trabajo de Hammer (1904: 61) se recogen casos semejantes en los que un complemento del predicado principal se une con un participio, como ocurre, por ejemplo,

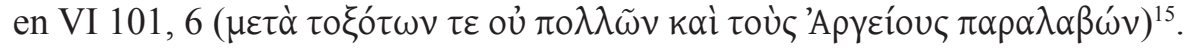

3. Volviendo a nuestro comentario: en la primera frase los dos participios en

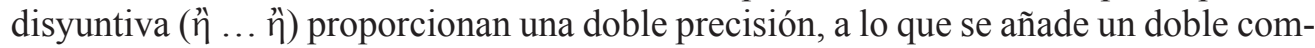
plemento indirecto unido por $\tau \varepsilon . . . k \alpha i ́$, «para mí y para los otros que me informaban». La tercera frase tiene dos precisiones negativas oủk... oủdé..., que recogen respec-

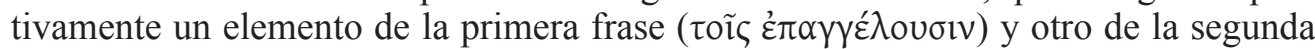

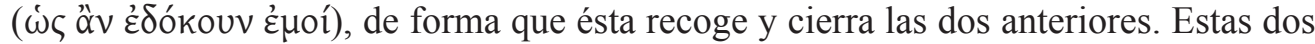
precisiones negativas hacen esperar dos positivas. Indudablemente ésas son los dos

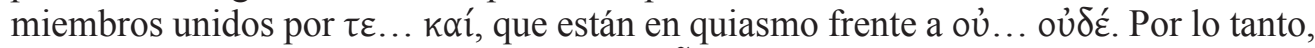

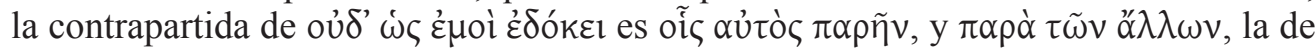

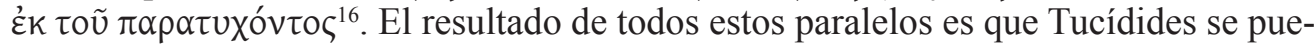
de permitir la omisión de todos los elementos que son obvios en la frase:

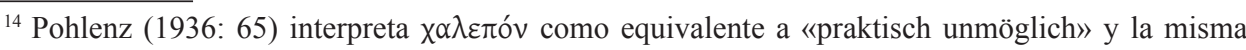
interpretación se encuentra en Tsakmakis (1998: 244, nota 17), que remite asimismo a Classen - Steup (1919: I 63), pero esta interpretación reduce el sentido del término griego que se explica teniendo en cuenta la capacidad de recordar que es propia de las personas analfabetas.

${ }^{15}$ Ros (1968: 393-394) comenta este ejemplo de variatio y aduce el paralelo de III 48, 2, donde se coordina mediante la disyuntiva tario de Poppo (1987), que considera la oración de relativo equivalente a un participio: Duo membra

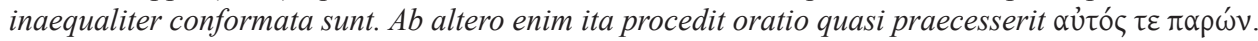

${ }^{16}$ Esta contraposición hace que entendamos el participio como masculino con la mayoría de los autores, aunque el testimonio de Dionisio de Halicarnaso $(6,822)$ haga de él un neutro, cf. Wille (1965:

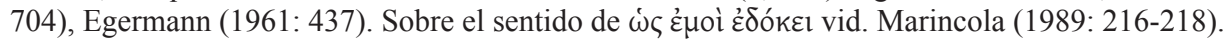




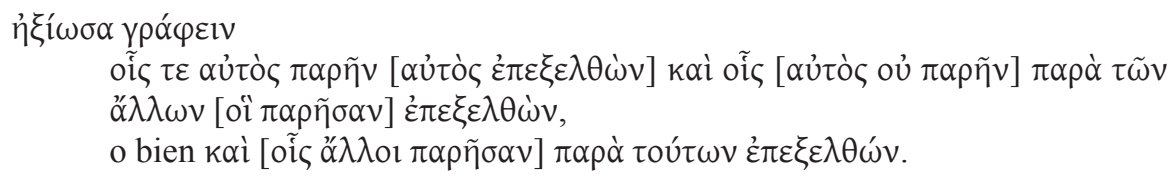

«Estimé escribirlos informándome no por quien tuviera más a mano, ni según me parecía a mí, sino siguiendo cada uno de los que fui testigo presencial y rastreando minuciosamente en la medida de lo posible los que no presencié mediante otros testigos.»

Nótese que $\dot{\pi} \pi \varepsilon \xi \xi \lambda \theta \omega ́ v$ requiere traducciones diversas en ambos miembros de la frase, pero el significado ${ }^{17}$ del verbo, «salir en busca de» (el enemigo), «perseguir», es válido en ambos casos. De todas formas en este contexto el verbo se usa metafóricamente. Su significado propio es «perseguir en acción de guerra, caza o juicio», con una asociación bien conocida en griego, y así lo usa Tucídides en la mayoría de los casos que recoge Bétant; en la mayor parte de ellos el verbo tiene un matiz de perseverancia en la acción, que a mi modo de ver, está también presente en este lugar. El paso de estos contextos al ámbito de la escritura o la "exposición» de un argumento sólo

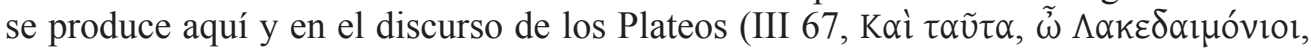

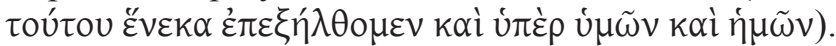

Tucídides en este caso busca insistentemente ${ }^{18}$ la precisión, en la medida de lo posible, según sus propias palabras, tanto en los acontecimientos en los que él mismo ha estado presente, como en los que ha tenido que recurrir a otros testigos presenciales. Este es el sentido de la última frase, si se tiene en cuenta el paralelo de la primera, tal

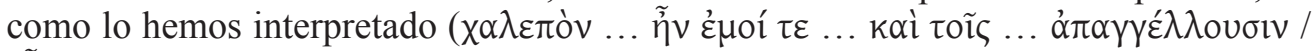

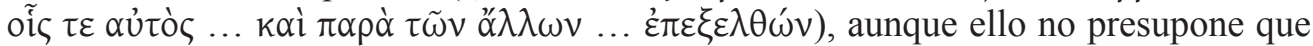
Tucídides haya hecho averiguaciones entre otros para fijar los acontecimientos que presenció en persona.

Sin embargo, a pesar de la objeción de Maddalena con sus antecedentes, no parece descartable la traducción «y mediante otros testigos presenciales», implicando que incluso en los hechos en los que él estuvo presente no se fió de su experiencia, sino que la contrastó con la de otros, sobre todo porque acaba de notar la dificultad de llegar a la verdad y, en calidad de general, Tucídides debía tener muy claro que, al menos en las batallas, todo lo que ocurre no se puede percibir desde un punto de vista único, sino que la realidad ha de perseguirse para lograr conocerla. Esto se entiende así sobre todo si se tiene en cuenta la frase que sigue inmediatamente a éstas, en la que se insiste y precisan las dificultades que hay para obtener una versión fiable de los hechos

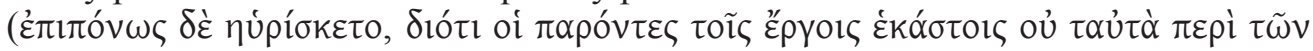

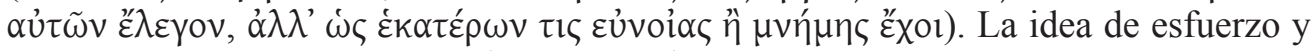

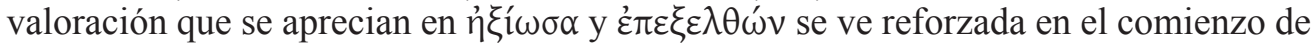

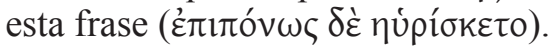

${ }^{17}$ Vid. Bétant (1843: 361).

${ }^{18}$ Nótese que este matiz se refuerza en la primera palabra de la frase siguiente: غ̇лı

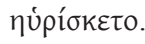




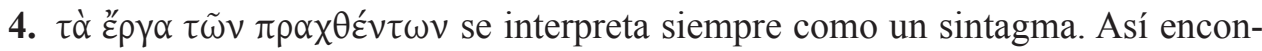
tramos las siguientes traducciones:

«I fatti compiuti durante la guerra», Maddalena (1951: 84).

«en ce qui concerne les actes qui prirent place au cours de la guerre», Romilly (1964: 14-15) ${ }^{19}$.

En la forma que adopta este sintagma juega con los dos participios pasivos en genitivo que le preceden:

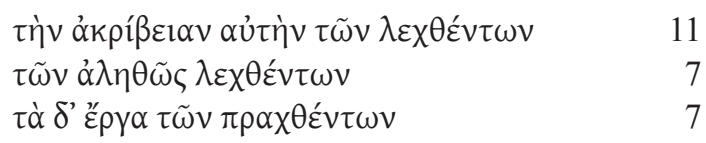

Este último sintagma parece plenamente redundante; evidentemente todo ع́pyov ha sido realizado y la posición predicativa del genitivo hace que debamos interpretarlo como partitivo, es decir, un genitivo «del todo», que es un uso raro ${ }^{20}$, o un genitivo de inherencia, que es un uso poético ${ }^{21}$. En ese caso, ¿cuáles son los «hechos» de «lo llevado a cabo»?; tal como apunta Classen (1966: 78) habría que entenderlo a partir

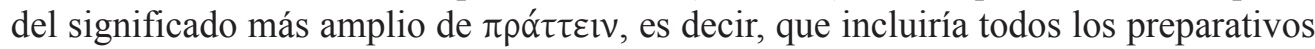
y asuntos relacionados con la guerra, pero la comparación con lo que dice Tucídides

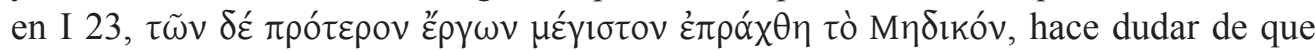
esta interpretación sea correcta. En cualquier caso, aún aceptando la explicación de Classen queda poco claro a qué se refiere ع́p $p \alpha$, si es que Tucídides quiere aislar de todos los acontecimientos relacionados con ella los hechos militares (genitivo partitivo) o hemos de entenderlo como una construcción pleonástica (genitivo de inherencia). Sea de ello lo que fuere, este giro constituye una llamada de atención sobre el texto a través de su expresión, si entendemos, como se hace generalmente, que el participio

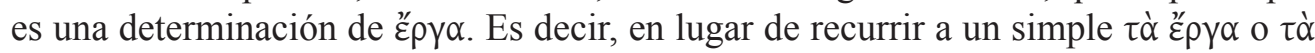
$\pi \rho \alpha \chi \theta \varepsilon \dot{v} v \tau \alpha$ Tucídides prefiere una expresión redundante ${ }^{22}$. El problema es determinar qué se quiere indicar con ella y por qué se ha elegido una determinación en genitivo.

El homeoteleuton y el ison, que vemos en los tres sintagmas anteriores, sirven para marcar la contraposición del plano de la acción de guerra con el plano del sentido de

${ }^{19}$ Siguen la misma interpretación Rodríguez Adrados (1967: I 106, «y en cuanto a los acontecimientos que tuvieron lugar en la guerra»), Macía (1989: 62, «Respecto a los hechos que tuvieron lugar en la guerra») y Torres Esbarranch (1990: 163, «Y en cuanto a los hechos acaecidos en el curso de la guerra»).

${ }^{20}$ Cf. Lasso de la Vega 1968: 437-438), aunque hay ejemplos en el propio Tucídides (cf. oi $\pi \alpha \lambda \alpha$ เoi $\tau \tilde{\omega} v \pi$ roı $\tau \tilde{\omega} v$, I 5, 2. Así lo interpreta Steup (1966: ad loc.).

${ }^{21}$ Vid. Lasso de la Vega (1968: 468-472) y Rodríguez Adrados ( 1992: 138-139). Sería en este caso sinónimo de ع̌p $\gamma \propto$ y equivalente a una aposición, cf. Kühner - Gerth (1898: II, 1, § 402 d).

${ }^{22}$ Tal como señala Gomme (1966: 139) es «somewhat clumsy phrase» y ve que ésta se ve forzada por el contexto y la línea de argumentación de Tucídides para demostrar que esta guerra es la más importante de todas, si se atiende a las acciones de guerra. 


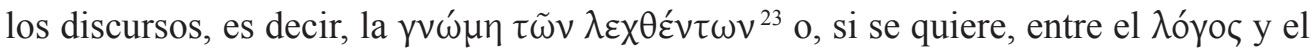
Épyov, como señaló ya Pohlenz (1919: 117) ${ }^{24}$. Pero, hay que notar que el contraste no está equilibrado, sino que se da entre el dativo singular indeterminado $\lambda{ }^{\prime} \gamma \omega$ y el

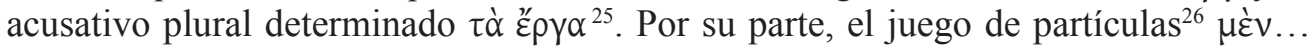

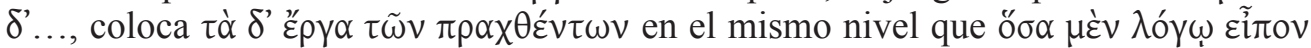

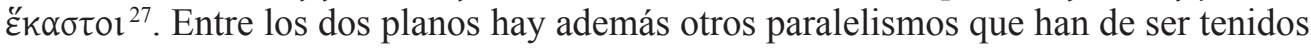
muy en cuenta: así en lo que se refiere, tanto a los discursos, como a los hechos, se

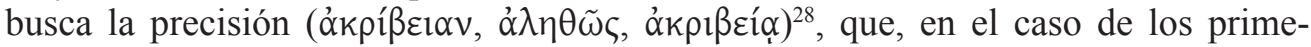

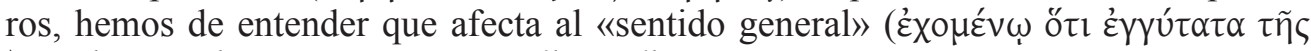

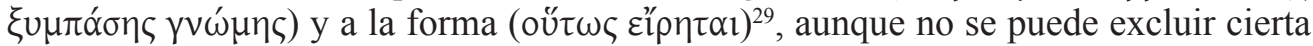
reelaboración por parte del autor ( $\delta \delta o ́ k o u v \mu o t)$, debida a la dificultad que entraña recordar lo que se ha dicho, tanto para el propio Tucídides, como para los demás testigos

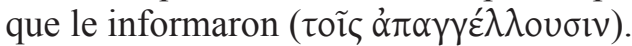

En cambio, en lo que se refiere a las acciones de guerra ( $\left.\varepsilon^{\rho} \rho \gamma \alpha\right)$ esta carga de subjetividad y reelaboración se descarta mediante dos oraciones negativas (oủk... oủ $\delta$ É...),

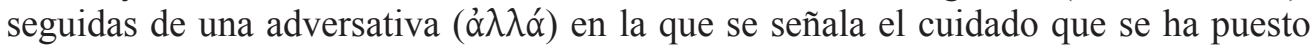
para averiguar los detalles. Este cuidado se subraya en la primera oración ( $\grave{\eta} \xi^{\prime}(\omega \sigma \alpha$

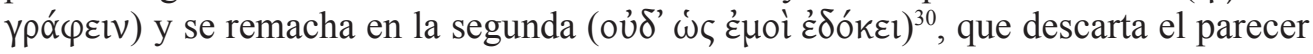
del autor a la hora de reflejar los hechos de guerra.

La importancia de los paralelos y contrastes que hemos visto anteriormente, se pone de manifiesto a la hora de determinar el significado de cada uno de los elementos

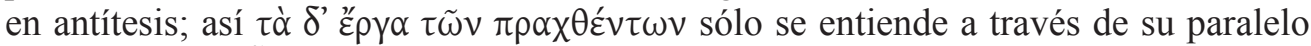

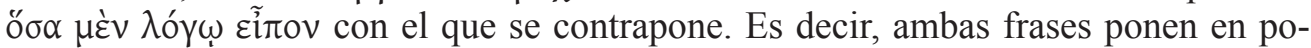
sición enfática los dos aspectos más relevantes a la hora de escribir la historia, los discursos y las acciones de guerra, pero cambiando por completo la sintaxis: ǒ $\sigma \alpha$ es un relativo que funciona como acusativo interno de $\varepsilon \tilde{i} \pi$ ov y queda casi suelto (cf. $\tilde{\omega} v$

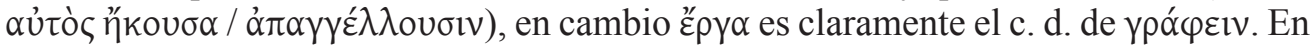
otras palabras, la contraposición de ambas frases con una estructura semejante y con

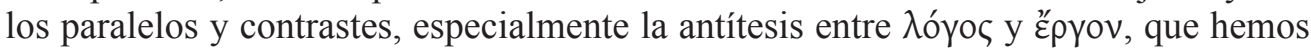

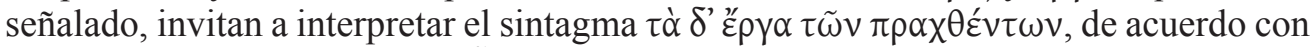

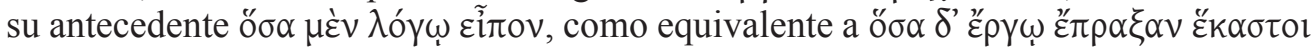

${ }^{23}$ Sobre el significado de este sintagma y los problemas que plantea su interpretación vid. Schmid (1954: 228-231).

${ }^{24}$ Cf. también Grosskinsky (1936: 249), Schmid (1954: 221 y 223) y Wille (1968: 700-701).

${ }^{25}$ Este plural se repite inmediatemente antes y después de las frases que estamos estudiando, de forma

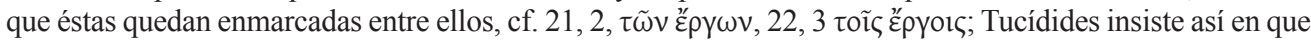
se trata de hechos de guerra cuya importancia justifica la propia importancia de esta guerra.

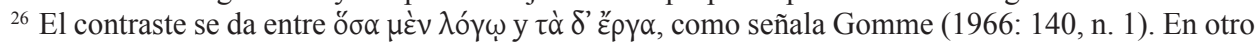
nivel estaría el primer $\delta \varepsilon ́$ que tiene una valor ilativo tal como lo interpreta Garrity (1998: 367).

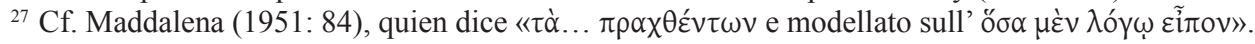

${ }^{28}$ Sobre la precisión que pretende Tucídides vid. Pohlenz (1936: 65-69), Wille (1965: 705) y en general Kuhn (2014).

${ }^{29}$ Como quiere interpretarlo Garrity (1998), cf. Maddalena (1951: 83-84).

${ }^{30}$ Cf. Harrison (1937: 7). Sobre el problema de la subjetividad implícita en esta frase vid. Schmid (1954: 224), quien remite a Patzer (1937: 37). 


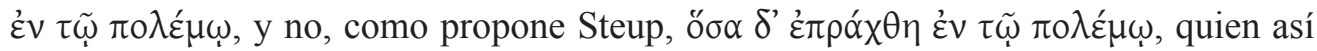
evita el problema que plantea la presencia de $\varepsilon^{\prime} p \gamma \alpha$.

«Cuanto dijeron de palabra ${ }^{31} \ldots$, cuanto hicieron de hecho», o bien «cuantas ideas dijeron de palabra..., cuantas acciones llevaron a cabo de hecho».

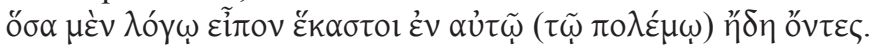

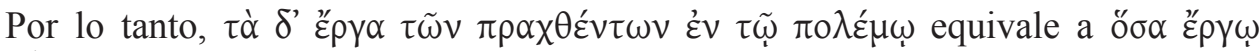

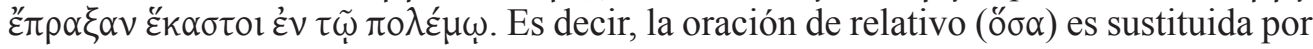
una determinación en genitivo formada por un participio pasivo (se requiere la pasiva por el carácter subjetivo del genitivo) ${ }^{32}$. De esta forma Tucídides fuerza un nuevo

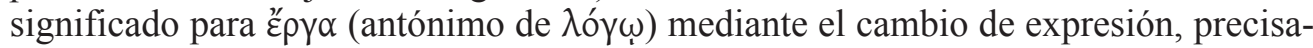
mente por su contraposición a $\gamma v \omega ́ \mu \eta \tau \tilde{\omega} v \lambda \varepsilon \chi \chi \theta \varepsilon ́ v \tau \omega \nu$.

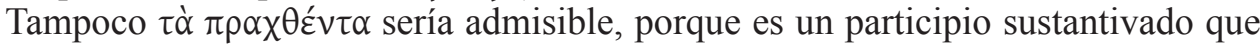
borraría la antítesis con $\gamma v \omega ́ \mu \eta$.

Partiendo de este paralelismo podemos suponer una transformación doble:

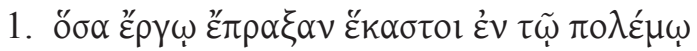

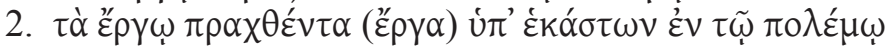

Pero, ö $\alpha$ es acusativo interno abreviado, que no puede suponer $\pi \rho \alpha ́ \gamma \mu \alpha \tau \alpha$ por su significado peyorativo, sino हैpyo «hazañas», «hechos de guerra». En la siguiente transformación aparece el sustantivo omitido épya, pero con el significado del complemento circunstancial $\varepsilon^{\prime} p(\omega$. Para adquirir este significado en lugar de las determi-

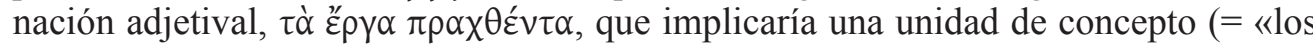
hechos realizados») $)^{33}$, Tucídides construye una determinación en genitivo $\tau \dot{\alpha}$ है $p \gamma \alpha \tau \tilde{\omega} v$ $\pi \rho \propto \chi \theta \varepsilon ́ v \tau \omega v$, conservando así la independencia de ambos conceptos: «las realidades de los hechos de guerra», o «los acontecimientos de los hechos de guerra», lo que, desde la perspectiva de su argumentación general (la Guerra del Peloponeso es la más importante de todas las habidas, tanto por las batallas, como por los medios desplegados en ella), resulta plenamente coherente. Pero, no podemos dejar de lado el hecho de que esta interpretación es demasiado compleja, porque parece requerir un análisis detallado de la estructura o la forma de lo que se está diciendo. Incluso para el estilo de Tucídides ésta resulta un tanto excesiva.

Frente a todo ello hay otra solución de los problemas que plantea esta frase, que no ha sido planteada por ningún comentarista. Tucídides puede estar jugando con el régimen de los verbos: el verbo $\pi v v \theta \alpha ́ v o \mu \alpha ı$ se construye tanto con acusativo c. d. de $\operatorname{cosa}^{34}$, como con genitivo complemento de $\operatorname{cosa}^{35}$. En otras palabras, $\tau \tilde{\omega} v \pi \rho \alpha \chi \theta \varepsilon ́ v \tau \omega \nu$

${ }^{31}$ Para el significado de $\lambda o ́ \gamma \omega$ en este lugar vid. Garrity (1998: 367-373).

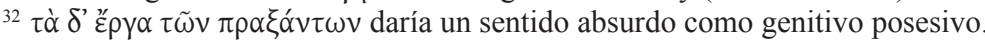

${ }^{33}$ Así parece entenderlo Romilly (1964: 14-15) al traducir «les actes qui prirent place au cours de la guerre».

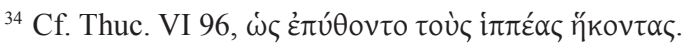

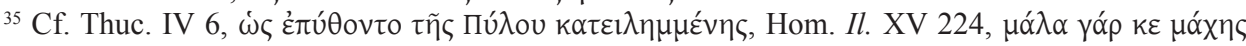

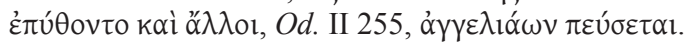




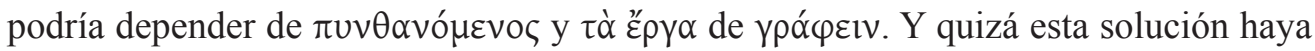
de ser tenida muy en cuenta. La antítesis se produciría, entonces, entre toù $\lambda$ óyous,

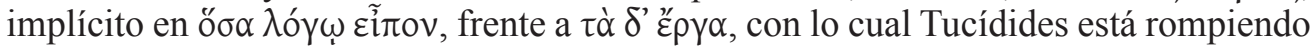
en la sintaxis el homeoteleuton $\lambda \varepsilon \chi \theta \dot{\varepsilon} v \tau \omega v / \pi \rho \alpha \chi \theta \dot{v} v \tau \omega v$, lo que es un recurso muy frecuente en él. En este caso la traducción quedaría así:

«Valoré escribir las acciones (bélicas), no informándome de lo acontecido en la guerra a partir del primer llegado, sino escrutando aquellas en las que estuve presente en persona y en la medida de lo posible indagando minuciosamente acerca de cada una por medio de los demás (testigos).»

Esta solución tiene en contra, aparentemente, la ruptura de la antítesis entre $\lambda o ́ \gamma o \varsigma$ y ع́pyov que, como hemos visto constituye el armazón argumental de todo el párrafo, pero hay que tener presente que hay cierta distancia entre el singular indeterminado

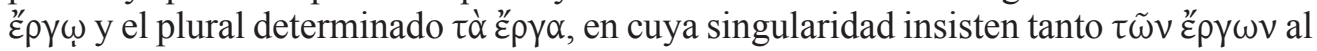

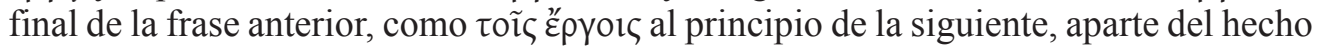
de que los paralelismos formales no tienen por qué coincidir con el significado de la frase, como señala Wille (1968: 648-692). Además esta solución supone un hipérbaton llamativo, cosa que no carece de paralelos en Tucídides. Casos semejantes a éste se encuentran en el libro I, como ocurre en el capítulo 70 de forma no demasiado exagerada

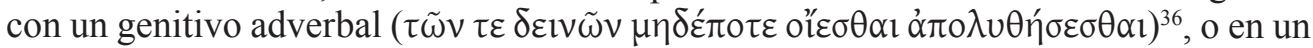

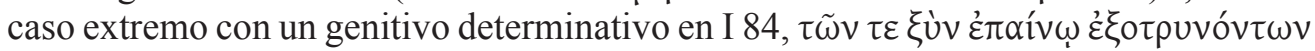

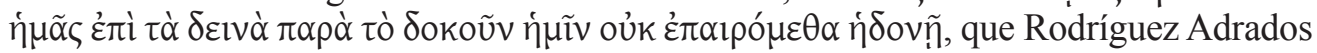
(1967: 163) traduce así: «no nos dejamos mover por el placer producido por los que nos incitan con su adulación a los peligros contra nuestro modo de pensar».

En cualquier caso es de notar que cualquiera de las interpretaciones que se quiera aceptar para esta frase no contradice su estructura general basada en la antítesis entre $\lambda o ́ \gamma o \varsigma$ y źpyov. A partir de este comentario creo que resulta claro que la solución más sencilla se encuentra en una línea distinta a la más evidente.

\section{BIBLIOGRAFÍA}

AlBerti, I. B. (1972), Thucydidis Historiae, Roma: Officina polygraphica.

Benedetti, F., Grandolini, S. (eds.) (2003), Studi di filologia e tradizione greca in memoria di Aristide Colonna, Perugia.

BétAnt, E. A. (1843), Lexicon Thucydideum, Ginebra: Carey.

Bicknell, Peter (1990), «Thucydides, I, 22: a provocation», $A C$ 59: 172-178.

Bonelli, Guido (2003), «Tucidide 1, 22, 1», en Benedetti - Grandolini (2003: 155-164).

Chantraine, P. (1968), Dictionnaire étymologique de la langue grecque, Paris: Klincksieck.

Classen, J., Steup, J. (1966), Thukydides, Berlin 1919-1922: Weidmann.

${ }^{36}$ Cf. I 72.1, I 79.2, I 36. 3, I 88. 1. Sobre el hipérbaton en Tucídides vid. Rittelmeyer (1915: 72-79) (non vidi). 
Dover, K. J. (1988), «Thucydides "as history" and "as literature"», The Greeks and their legacy, II: 53-64, Oxford.

Egermann, F. (1961), «Zum historiographischen Ziel des Thukydides», Historia 10: 435-447.

Flaschar, Helmult, Gaiser Konrad (eds.) (1965), Synusia. Festgabe für Wolfgang Schadewaldt, Pfullingen.

FinLey, J. H. Jr. (1942), Thucydides, Cambridge: HUP.

GARrity, Thomas Francis (1998), «Thucydides 1. 22. 1: content and form in the speeches», AJPh 119: 361-384.

Golan, D. (1976-1977), «Some comments on Thucydides 1.20-23», SCI 3: 1-7.

Gomme, A. W. (1937), Essays in Greek history and literature, Oxford: Blackwell.

- (1966), A historical commentary on Thucydides I, Oxford: Clarendon.

Grosskinsky, A. (1936), Das Programm des Thukydides, Berlin: Junker und Dünnhaupt.

Harrison, A. R. W. (1937), «Thucydides 1, 22», CR 51: 6-7.

Herter, H., (ed.) (1968), Thukydides, Darmstadt: Wiss. Buchgesellschaft.

HuArt, P. (1968), Le vocabulaire de l'analyse psychologique dans l'oeuvre de Thucydide, Paris: Klincksieck.

Hornblower, S. (1988), Thukydides, London.

KÜHNER, R.; GERTH, B. (1898), Ausführliche Grammatik der griechischen Sprache, I-II, Leipzig.

Iglesias Zoido, J. C. (1989): «Acercamiento a la polémica sobre Tucídides I, 22, 1», Anuario de estudios filológicos (Cáceres Universidad de Extremadura), 12: 125-132.

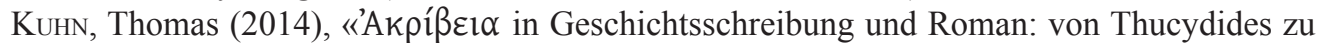
Chariton», Gymnasium 121: 131-153.

Lasso de la Vega, José (1968), Sintaxis griega, Madrid: CSIC.

LoRIAux, R. (1982), «Les discours de Thucydide (I, 22)», LEC 50: 289-292.

Luschnat, O. (1970), «Thucydides der historiker», RE Suppl. XII: 1085-1354.

Macía, L. (1989), Tucídides. Historia de la guerra del Peloponeso, Madrid: Akal.

Maddalena, A. (1951), Thucydidis Historiarum, Firenze: La Nuova Italia.

Marincola, J. M. (1989), «Thucydides 1.22.2», CPh 89: 216-223.

Parry, A. M. (1981), Logos und ergon in Thudydides, New York: Arno Press.

Patzer, Harald (1937), «Das Problem der Geschichtsschreibung des Thukydides und die thukydideische Frage», Neue deutsche Forschungen 6: 37.

Pohlenz, M. (1919), «Thukydidesstudien», Nachr. Ges. Wiss. zu Göttingen, 1919: 95-138.

- (1936), «Die Thukydische Frage in Lichte der neueren Forschung», GGA 198: 281-300, (= HERTER 1968: 59-81).

Poppo, E. F.; Stahl, I. M. (1987), Thucydides de Bello Peloponnesiaco libri octo, New York: Garland.

Rittelmeyer, F. (1915), Thukidides und die Sophistik, Borna-Leipzig.

Romilly, J. de (1964), Thucydide, París: Belles Lettres.

Rodríguez Adrados, Francisco (1967), Tucídides. Historia de la guerra del Peloponeso, Madrid: Hernando.

- (1992), Nueva sintaxis del griego antiguo, Madrid: Gredos.

Ros, J. (1968), Die Metabolé (variatio) als Stilprinzip des Thukydides, Paderborn 1938.

Ruıgh, C. (1971), Autour de «Te épique», Amsterdam: Hakkert.

Schadewaldt, W. (1929), Die Geschichtsschreibung des Thukydides, Berlin: Weidmann 1971.

Schmid, Walter (1954), «Zu Thucydides, I, 22, 1 und 2», Philologus 99: 220-233. 
Stadter, Ph. A. (1973), The speeches in Thucydides, Chappel Hill: U. N. Carolina.

Swain, S. (1993), «Thukydides 1. 22. 1 and 3. 82. 4», Mnemosyne 46: 33-45.

Tsakmakis, Antonis (1998), «Von Rhetorik zur Geschichtsschreibung: Das Methodenkapitel des Thukydides», RhM 141: 239-255.

West, W. C. III (1973), «A bibliography or scholarship on the speeches in Thukydides», en STADTER (1973: 124-165).

Wille, Günther (1965), «Zu Stil und Methode des Thukydides», en Flaschar - Gaiser (1965: 53-77), (= HERTER, 1968: 683-716).

Wilson, J. (1982), «What does Thucydides claim for his speeches?», Phoenix 36: 95-104. 